\title{
Strategic Interaction of State Institutions and Enterprises with Economic Security Positions in Digital Economy
}

\author{
YEVHENII RUDNICHENKO ${ }^{1}$, STEPAN MELNYK ${ }^{2}$, NATALIIA HAVLOVSKA ${ }^{1}$, \\ OLENA ILLIASHENKO ${ }^{3}$, NATALIA NAKONECHNA ${ }^{4}$
}

\author{
${ }^{1}$ Department of Management, Administration and Hotel and Restaurant Business, Khmelnitsky \\ National University, UKRAINE \\ ${ }^{2}$ Faculty №2, Lviv State University of Internal Affairs, UKRAINE \\ ${ }^{3}$ Department of financial-economical security, accounting and audit, O.M. Beketov National \\ University of Urban Economy, UKRAINE \\ ${ }^{4}$ Department of Finance and Accounting, Lviv State University of Internal Affairs, UKRAINE
}

\begin{abstract}
The purpose of the study is to develop amodel of the influence of state institutions on the enterprise state institutions and enterprises - proposed indicators determine the strategy of the game participant and their behavior in such a game. The content of each of the proposed indicators is considered and it is shown how their value can be obtained. The proposed indicators for both participants in the game are leveled to a single scale. The indicators of the model of the antagonistic game between the enterprise and state institutions are determined that became the basis for the construction of the game model, which is a tuple. The developed model of the influence of state institutions on the enterprise economic security system, represented by a combination of possible strategies for the behavior of both participants in the game in hyper cubic five- dimensional space, which are expressed by the values of indicators for each of these participants, as well as the

calculated "gain" for each of these combinations. Using this model allows to achieve different results depending on the goal of the study. The main result of the model is the search for a balance of interests in the

"enterprise_state" system. That is, the search for those points of stable choice that can be obtained using the developed model, namely: search for a local or general extremum in the game field, which will determine the maximum win for one of the participants or the total win of two participants at the same time; research and quantitative estimation of the actual and maximum possible negative impact of state institutions on the economic security system of business entities; determining the expected change in the influence of state institutions on the economic security system of business entities due to changes in its strategy; the establishment of the best strategy for a business entity in existing conditions, which, according to the selected criterion, will ensure the stable functioning of the economic security system at an enterprise; determination of the limits of the negative impact of state institutions on the economic security system of an enterprise.
\end{abstract}

Key-Words: - economic security, government institutions, enterprise, modeling, game theory, scenario.

Received: August 16, 2021. Revised: November 29, 2020. Accepted: December 31, 2021. Published: January 14, 2021

\section{Introduction}

The quick reaction of the business environment to external stimuli requires an adequate estimation and modeling of possible scenarios and ways to improve the economic security of business entities. Modeling of the processes associated with the creation, functioning and development of enterprises, and accordingly with the formation of their economic security system, suggests the existence of a certain alternative choice of solution. After this an action or inaction comes, that leads to certain results. Moreover, the results may be known in advance, partially known or unknown.

Therefore, modeling the interaction of state institutions and business entities is very important, because it allows not only to determine the situation, but also to form scenarios for changing this situation, the reaction of all participants in it, etc. Appropriate modeling tools are used depending on the specifics of a choice. Simulation, mathematical modeling, descriptive models, prescriptive models, game theory, etc. are traditionally used modeling tools in constructing models of economic phenomena and processes.

The modeling task is to analyze the actual impact of state institutions on the economic security system of business entities with determining the results of this impact, finding the optimal strategy for the enterprise (according to certain optimality criteria), and formulating proposals for adjusting the influence of state institutions to achieve the desired behavior of 
business entities due to influence on the economic security system of these entities, as well as the construction of a forecast on the likely impacts of scenarios of behavior of participants of analyzing interaction.

Since the interaction of business entities and entities of state institutions occurs most often in conditions of uncertainty, such tools as fuzzy sets, utility theory, game theory can be used in modeling and, given the antagonistic nature and strategic orientation of the interaction of the above entities, the most suitable for modeling such an interaction is game theory, which some experts [31] interpret as a mathematical tool for modeling an alignment of parties interests.

Game theory as a modeling tool cannot be the best decision for solving the task, since there is clearly a conflict of interests of the parties, and the parties of such a conflict make strategic decisions often not knowing about the opponents. In addition, the balance of interests of the parties, which will satisfy agents in game theory from an economic point of view, is displayed as a zero result, that is, any of the agents will have no gain, and therefore no loss. This situation takes on signs of harmonization of relations between the subjects of interaction and is the most constructive.

\section{Problem Statement}

The purpose of the study is to develop a model of the influence of state institutions on the enterprise economic security system by using the provisions of game theory.

It is worth noting that a game with the participation of business entities and state institutions, at first glance, is purely antagonistic, since both participants are directly interested in various extremums of the outcome of the game - the business entity seeks to minimize the payments, and state institutions - to maximize them. But, if we consider the situation in more detail, then the interests of the game participants coincide in the long run. An optimal situation for business is when not only simple reproduction of means and objects of labor will be ensured, but a certain reserve for development will exist. And the position of the state lies precisely in the need to ensure the implementation of the revenue side of the budget, to ensure the growth of the employed population and to form reserves to overcome potential crisis phenomena. That is, in the long run, both business and a state are directly interested in business growth, but in the short run, there is a conflict of interests between a state and business, which requires balanced decisions aimed at harmonizing relations. And in this situation, one of the most effective tools for modeling possible situations and finding ways to solve the existing problems of the influence of state institutions on the enterprise economic security system is the theory of antagonistic games, which allows to quantitatively describe the antagonistic situation, find the most appropriate solution for all its participants, suggest possible scenarios, etc.

\section{Review of literature}

There are many views on the nature of game theory in literary research, namely: theory of rational behavior of people with conflicting interests [3]; science of strategic thinking [8]; mathematical study of strategy and conflict [6]; study of mathematical models of strategic interaction among rational decision-makers [17].

The first known discussion of game theory occurred in a letter believed to be written in 1713 by Charles Waldegrave, an active Jacobite and uncle to James Waldegrave, a British diplomat [5]. In 1913, Ernst Zermelo published Über eine Anwendung der Mengenlehre auf die Theorie des Schachspiels (On an Application of Set Theory to the Theory of the Game of Chess), which proved that the optimal chess strategy is strictly determined [33]. Game theory did not really exist as a unique field until John von Neumann published the paper On the Theory of Games of Strategy in 1928 [22]. His paper was followed by his 1944 book Theory of Games and Economic Behavior co-authored with Oskar Morgenstern [16]. Also, among the founders of the above-mentioned theory are such Nobel Prize winners in economics 1994 as John Nash, Reinhard Selten, John Harsanyi [10], [19], [20]. In 2005, game theorists Thomas Schelling [26], [27] and Robert Aumann [2], [4] followed Nash, Selten, and Harsanyi as Nobel Laureates. Schelling worked on dynamic models, early examples of evolutionary game theory. In 2007, Leonid Hurwicz [13], Eric Maskin [15], and Roger Myerson [17], [18] were awarded the Nobel Prize in Economics "for having laid the foundations of mechanism design theory". Myerson's contributions include the notion of proper equilibrium, and an important graduate text: Game Theory, Analysis of Conflict [17]. In 2012, Alvin E. Roth [23], [24] and Lloyd S. Shapley [28], [29] were awarded the Nobel Prize in Economics "for the theory of stable allocations and the practice of market design". In 2014, the Nobel went to game theorist Jean Tirole [32].

Game theory in the form known to economists, social scientists, and biologists, was given its first 
general mathematical formulation by John von Neuman and Oskar Morgenstern [21]. As [11], [14], [25] noted in the study of economic systems, complex mathematical tools that allow predicting options for the development of events, determining scenarios and substantiate strategic decisions are more and more often used.

Game theory is actively used in modern research to solve various problems of practical orientation. Its apparatus can be used to analyze situations in order to make strategic decisions, competitiveness, "balance of interests", measure the level of risk and uncertainty, etc. Recent research includes the following: E. Adida and V. DeMiguel [1] conduct research on multi-production and retail chains; J. Chen and B. Chen [7] study the optimal policy of consumer involvement in competition; M. Esmaeili and all [9] study the optimal pricing solutions in closed supply chain systems; B. Shen and all [30] study the problem of coordination of the supply chain of two products; Zlotenko O. and all [34] study this issue in order to optimize the structure of funding sources; M. Hua and all [12] investigate the system of return channels using game theory.

\section{Materials and methods}

It is possible to determine the balance of interests of economic entities and the state on the basis of game theory methods, which allow to find the optimal correlation of the above indicators. These ratios will maximize the harmony of the interests of a state and an enterprise.

In this situation, there are two parties involved in the game model - the state and the enterprise. As already noted, the state has three leverage - the tax burden ratio $x_{1}$, the indicator of tax impact turbulence $x_{2}$ and the indicator of estimation of operational processes of state institutions ratio $x_{3}$. We will group these variables into a pure strategy (1) of the first player, who will personify a state:

$x=\left[x_{1} x_{2} x_{3}\right] \in S \subset R^{3}$

The location (ranking) of the component point (1) is made in accordance with their numbers and does not have fundamental significance. The set $S$ as a subset of three-dimensional Euclidean space is the set of all pure state strategies. This set consists only of admissible (legal) three-element solutions that can be established at the state level. Under the aforementioned conditions, the normalization of the tax burden ratio, the indicator of tax impact turbulence and indicator of estimation of operational processes of state institutions, the set of pure state strategies is a single cube:
$S=\prod_{k=1}^{3}[0 ; 1] \subset R^{3}$

A pure strategy of the second player, which will represent the business enterprise, consists of two components - variables $y_{1}$ (indicator characterizing the use of shadow operations by an enterprise) and $y_{2}$ (indicator characterizing the forced additional costs to protect the business from the negative impact of a state). This pure strategy (3) as a point of two-dimensional Euclidean space represents the choice of a business entity:

$y=\left[y_{1} y_{2}\right] \in E \subset R^{2}$

The set $E$ as a subset of two-dimensional Euclidean space contains all possible or permissible choices of an enterprise. According to the above conditions for normalizing variables $y_{1}$ and $y_{2}$, many of an enterprise pure strategies are a single square:

$E=\prod_{j=1}^{2}[0 ; 1] \subset R^{2}$

Thus, the game model is a tuple:

$\langle S, E, P(x, y)\rangle=\left\langle S, E, P\left(x_{1}, x_{2}, x_{3}, y_{1}, y_{2}\right)\right\rangle$

where the function of five variables $P(x, y)$ characterizes the gain (level of utility) received by a state in the situation $\{x, y\}$, when it used the strategy $x \in S$ in (1), and an enterprise used the strategy $y \in E$ in (3).

Function $P(x, y)$ describes the losses (costs) of an enterprise in the situation $\{x, y\}$. This function is set on a single hypercube:

$$
S \times E=\prod_{k=1}^{3}[0 ; 1] \times \prod_{j=1}^{2}[0 ; 1]=\prod_{l=1}^{5}[0 ; 1] \subset R^{5}
$$

In game (5) with its kernel $P(x, y)$ on a single hypercube (6) the set of all situations in pure strategies is a continuum of pairs $\{x, y\}$ of points of a single hypercube (6).

Game (5) will be solved either in pure strategies $\left\{x^{*}, y^{*}\right\}$, or in mixed $\left\{r^{*}(x), q^{*}(y)\right\}$, where $q^{*}(y)$ is the probability distribution over the set $E$. So, the main question is the search (estimation) of the kernel $P(x, y)$ of game (5) on a single hypercube (6).

With fixed variables $y_{1}$ and $y_{2}$ the state gain, the normalized level of revenues to the state budget, will increase with an increase in one (or all at once) of components of the pure strategy (1). With fixed variables $x_{1}, x_{2}$ and $x_{3}$, the utility of the state will decrease with an increase in one variable or both. Thus, the kernel of the game (5) can be represented in multiplicative form: 
$P\left(x_{1}, x_{2}, x_{3}, y_{1}, y_{2}\right)=\left(\prod_{k=1}^{3} x_{k}^{\eta_{k}\left(x_{k}\right)}\right) \times\left(1-y_{1}\right)^{\mu_{1}\left(y_{1}\right)} \times$

$\times\left(1-y_{2}\right)^{\mu_{2} y_{2}}-\left(\prod_{k=1}^{3} x_{k}-x_{k}^{\langle\text {the reg. lim. }\rangle}\right)^{\mu_{k}\left(x_{k}\right)} \times$

$\times\left(y_{1}-y_{1}\langle\text { the reg.lim. }\rangle\right)^{\mu_{1}\left(y_{1}\right)}\left(y_{2}-y_{2}{ }^{\langle\text {the reg.lim. }\rangle}\right)^{\mu_{2}\left(y_{2}\right)} \times$

$\times\left(\min _{k=1,3}\left\{\operatorname{sign}\left(x_{k}-x_{k}{ }^{\langle\text {the reg. lim. }\rangle}\right)\right\}\right) \times$

$\times\left(\min \left\{\operatorname{sign}\left(y_{1}-y_{1}{ }^{\langle\text {the reg.lim. }\rangle}\right), \operatorname{sing}\left(y_{2}-y_{2}{ }^{\langle\text {the reg.lim. }\rangle}\right)\right\}\right)$,

in which $\eta_{k}\left(x_{k}\right)$ - impact estimation of $k$-th component of a state pure strategy in the game (1) for the state gain $P\left(x_{1} \cdot x_{2} \cdot x_{3} \cdot y_{1} \cdot y_{2}\right) ; x_{k}^{\text {〈thereg.lim〉. }}$ regulatory limit value of this component; $k=\overline{1,3}$; $\mu_{1}\left(y_{1}\right)$ - estimation of the negative impact force of a

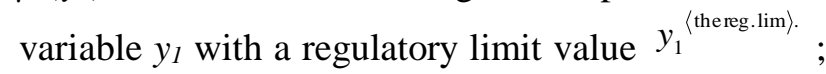
$\mu_{2}\left(y_{2}\right)$ - estimation of the force of the negative influence of a variable $y_{2}$ in game (5) with its regulatory limit value $y_{2}{ }^{\langle\text {thereg.lim〉. }}$ for the state gain $P\left(x_{1} . x_{2} . x_{3} \cdot y_{1} \cdot y_{2}\right)$.

We will present estimates of the influence force of each component of a state pure strategy in game (5) in multiplicative form with the known nominal value of the component taking into account the multiplication and adaptation coefficients of the respective component. So:

$$
\eta_{k}\left(x_{k}\right)=\eta_{k}^{\langle 0\rangle} K_{\text {mult }}^{\left\langle\eta_{k}\right\rangle} K_{\text {adap.sat. }}^{\left\langle\eta_{\eta}\right\rangle} K_{\text {adap.ex. }}^{\left\langle\eta_{\eta}\right\rangle} \text { with } k=\overline{1,3} \text {, }
$$

in which $\eta_{k}^{\langle 0\rangle}$ is the nominal value of the impact force of $k$-th component of a state pure strategy in the game (5) for the state gain $P\left(x_{1} \cdot x_{2} \cdot x_{3} \cdot y_{1} \cdot y_{2}\right), K_{\text {mult }}^{\left\langle\eta_{k}\right\rangle}$ is the multiplier of the component $x_{k}, K_{\text {adap.sat. }}^{\left\langle\eta_{k}\right\rangle}$ component adaptation ratio $x_{k}$ before saturation, and $K_{\text {adap.ex }}^{\left\langle\eta_{k}\right\rangle}$

- component adaptation ratio $x_{k}$ before exceeding an economic norm.

Definitely, in a first approximation (testing), the coefficient $K_{\text {mult }}^{\left\langle\eta_{k}\right\rangle}$ can be considered as a one:

$K_{\text {mult }}^{\left\langle\eta_{k}\right\rangle}=1 \forall k=\overline{1,3}$.

Adaptation component ratio $x_{k}$ before saturation, we will estimate in this way:

$K_{\text {adap.sat. }}^{\left\langle\eta_{k}\right\rangle}=1+x_{k}^{\text {Sadap.sat }\left(\eta_{k}\right)}{ }_{\text {при }} k=\overline{1,3}$

in which Sadap.sat. $\left(\eta_{k}\right)$ is the value of the adaptation force of $k$-th component of a state pure strategy in the game (5) before saturation.

The value of the estimation of component adaptation ratio $x_{k}$ before exceeding the economic norm depends on the normative threshold value $x_{k}{ }^{\text {〈thereg.lim〉. }}$ of this component and the value of the force of the difference impact between $x_{k}$ and $x_{k}{ }^{\text {the reg.lim }\rangle \text {. }}$.

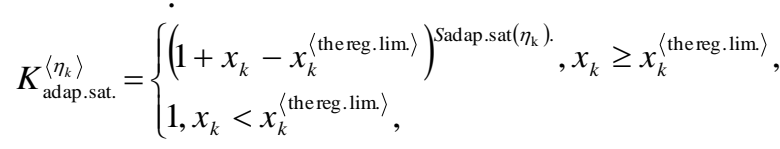

in which $K_{\text {adap.sat. is the value of the component }}^{\left\langle\eta_{k}\right\rangle}$ adaptation force $x_{k}$ before exceeding the economic norm.

Obviously, expression (11) can be represented in a more convenient form, without two-line curly braces:

$$
\begin{aligned}
& K_{\text {adap.sat. }}^{\left\langle\eta_{k}\right\rangle}=\left(1+x_{k}-x_{k}^{\text {Sadap.sat }\left(\eta_{k}\right)}\right)^{\operatorname{Sadap.sat}\left(\eta_{\mathrm{k}}\right)} \times \\
& \times \frac{1+\operatorname{sign}\left(x_{k}-x_{k}^{\langle\text {the reg.lim. }\rangle}\right)}{2}+ \\
& +\frac{1-\operatorname{sign}\left(x_{k}-x_{k}^{\langle\text {the reg.lim. }\rangle}\right)}{2} \text { with } k=\overline{1,3}
\end{aligned}
$$

The final estimation (8) of the impact force of the $k$-th component of a state pure strategy in the game (5) taking into account (9) - (12) will be written as following:

$$
\eta\left(x_{k}\right)=\eta_{k}^{\langle 0\rangle}\left(1+x_{k}^{\text {Sadap.sat }\left(\eta_{k}\right)}\right)\left[\begin{array}{l}
\left(1+x_{k}-x_{k}^{\langle\text {the lim.reg. }\rangle)^{\text {Sadap.sat }\left(\eta_{k}\right)}} \times\right. \\
\times \frac{1+\operatorname{sign}\left(x_{k}-x_{k}^{\langle\text {the reg. lim. }\rangle}\right)}{2}+ \\
+\frac{1-\operatorname{sign}\left(x_{k}-x_{k}^{\langle\text {thereg.lim. }\rangle}\right)}{2}
\end{array}\right]
$$

with $k=\overline{1,3}$

Estimations of the influence of variables $y_{1}$ and $y_{2}$ in game (5) we also present in multiplicative form with the known nominal values of these indicators, taking into account the respective multiplication and adaptation coefficients. Similarly to (8), we have:

$\mu_{j}\left(y_{j}\right)=\mu_{j}^{\langle 0\rangle} K_{\text {mult }}^{\left\langle\mu_{j}\right\rangle} K_{\text {adap.sat. }}^{\left\langle\mu_{j}\right\rangle} K_{\text {adap.ex. }}^{\left\langle\mu_{j}\right\rangle}$ with $j=\overline{1,2}$.

In formula (14) $\mu_{j}^{\langle 0\rangle}$ is the nominal value of the force of the (negative) influence of the variable $y_{1}$, and $\mu_{2}^{\langle 0\rangle}$ - the nominal value of the force of the (negative) influence of the variable $y_{2}$ in game (5) for the state gain $P\left(x_{1}, x_{2}, x_{3}, y_{1}, y_{2}\right) ; K_{\text {mult }}^{\left\langle\mu_{j}\right\rangle}$ and $K_{\text {mult }}^{\left\langle\mu_{2}\right\rangle}-$ coefficients of multiplication of ratios of variables $y_{1}$ and $y_{2}$ accordingly; $K_{\text {adap.sat. }}^{\left\langle\mu_{j}\right\rangle}$ and $K^{\left\langle\mu_{2}\right\rangle}$

$K_{\text {adap.sat. }}$ - coefficients of adaptation of variables $y_{1}$ and $y_{2}$ before saturation, respectively; $K_{\text {adap.ex. }}^{\left\langle\mu_{j}\right\rangle}$ and $K_{\text {adap.ex. }}^{\left\langle\mu_{2}\right\rangle}$ - adaptation coefficients of the variable indicators $y_{1}$ and $y_{2}$ before exceeding the economic 
norm, respectively.

As in (9), in the first approximation (testing), the coefficients $K_{\text {mult }}^{\left\langle\mu_{j}\right\rangle}$ and $K_{\text {mult }}^{\left\langle\mu_{2}\right\rangle}$ can be considered as a one:

$K_{\text {mult }}^{\left\langle\mu_{j}\right\rangle}=1, K_{\text {mult }}^{\left\langle\mu_{2}\right\rangle}=1$

Coefficients of adaptation of variables $y_{1}$ and $y_{2}$ before saturation, we estimate similarly to the estimates (10):

$$
K_{\text {adap.sat. }}^{\left\langle\mu_{j}\right\rangle}-1+y_{j}^{\text {Sadap.sat }\left(\mu_{\mathrm{j}}\right)} \text { with } j=\overline{1,2} \text {. }
$$

in which Sadap.sat. $\left(\mu_{1}\right)$ and Sadap.sat. $\left(\mu_{2}\right)$ are values of the force of adaptation of variables $y_{1}$ and $y_{2}$ in game (5) respectively.

As expected in relation (11), the values of the estimates of adaptation coefficients $K_{\text {adap.sat. }}^{\left\langle\mu_{1}\right\rangle}$ and $K_{\text {adap }}^{\left\langle\mu_{2}\right\rangle}$

$K_{\text {adap.sat. before exceeding the economic norm of }}^{\left\langle\mu_{2}\right\rangle}$ variables $y_{1}$ and $y_{2}$ depend on their normative limit values $y_{1}^{\text {Sadap.sat }\left(\mu_{\mathrm{j}}\right)}$ and $y_{2}^{\text {Sadap.sat }\left(\mu_{\mathrm{j}}\right)}$ respectively, as well as on the values of force of influence of differences $y_{1}-y_{1}{ }^{\text {thereg. lim. }}$ and $y_{2}-y_{2}{ }^{\text {thereg. lim. }}$ :

$K_{\text {adap.sat. }}^{\left\langle\mu_{j}\right\rangle}=\left(1+y_{j}-y_{j}{ }^{\langle\text {the reg.lim. }\rangle}\right)^{S \text { Sadap.sat }\left(\mu_{j}\right)} \times$

$\times \frac{1+\operatorname{sign}\left(y_{j}-y_{j}^{\langle\text {the reg. lim. }\rangle}\right)}{2}+$

$+\frac{1-\operatorname{sign}\left(y_{j}-y_{j}^{\langle\text {the reg. lim. }\rangle}\right)}{2}$ with $j=\overline{1,2}$,

in which Sadap.sat. $\left(\mu_{1}\right)$ and Sadap.sat. $\left(\mu_{2}\right)$ are values of the force of adaptation of variables $y_{1}$ and $y_{2}$ respectively before exceeding the economic norm.

Final assessment of the influence of variables $y_{1}$ and $y_{2}$ of an enterprise in game (5), taking into account (15) - (17), we write as following:

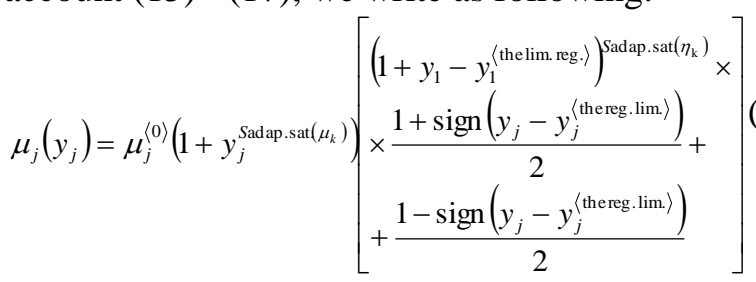

with $j=\overline{1,2}$

\section{Results}

The normative limit values of the components in a state pure strategy (1) based on expert observations, we set as:

$$
\begin{aligned}
& x_{1}^{\langle\text {the reg. } . \text { lim }\rangle}=x_{2}^{\langle\text {the reg. } . \text { lim }\rangle}=0.6 \sqrt{b^{2}-4 a c} \\
& \text { and }{ }^{x_{3}^{\langle\text {thereg. } . \text { lim }\rangle}}=0.8 .
\end{aligned}
$$

Normative limit values for variables $y_{1}$ and $y_{2}$ in its pure strategy (3), also based on expert observations, we set as:

$y_{1}^{\langle\text {thereg. lim }\rangle}=0.6$ and $y_{2}{ }^{\langle\text {therg. } . \text { lim }\rangle}=0.4$.

To determine the nominal values of the force of influence $\left\{\eta_{k}^{\langle 0\rangle}\right\}_{k=1}^{3}$ component of a state pure strategy and the power of negative influence $\mu_{1}^{\langle 0\rangle}$ of variable $y_{1}$ and the power of negative influence $\mu_{2}^{\langle 0\rangle}$ of variable $y_{2}$ of an enterprise for the state gain $P\left(x_{1}, x_{2}, x_{3}, y_{1}, y_{2}\right)$ in game (5) we use the method of hierarchy analysis. By the method of hierarchy analysis, we obtain the following matrix of pairwise comparisons:

$$
\mathrm{M}=\left(m_{l q}\right)_{5 \times 5}=\left(\begin{array}{ccccc}
1 & 3 & 5 & 3 & 1 \\
1 / 3 & 1 & 1 & 3 & 1 \\
0.2 & 1 & 1 & 1 & 1 / 3 \\
1 / 3 & 1 / 3 & 1 & 1 & 1 / 3 \\
1 & 1 & 3 & 3 & 1
\end{array}\right)
$$

that is made up of a sequence of elements (objects):

$\left\{\eta_{1}^{\langle 0\rangle}, \eta_{2}^{\langle 0\rangle}, \eta_{3}^{\langle 0\rangle}, \mu_{1}^{\langle 0\rangle}, \mu_{2}^{\langle 0\rangle}\right\}$

Accordingly, calculating geometric mean values:

$m_{1}=\left(\prod_{q=1}^{5} m_{l q}\right)^{0.2} \forall l=\overline{1,5}$

for the elements of sequence (22), we get: $m_{1}=4.5^{0.2}, m_{2}=1^{0.2}=1, m_{3}=15^{0.2}$, $m_{4}=27^{0.2}, m_{5}=9^{0.2}$.

Now, taking into account (24), point estimates (local priorities) of the nominal values of the influence force $\left\{\eta_{k}^{\langle 0\rangle}\right\}_{k=1}^{3}$ component of a state pure strategy (1) for the state gain $P\left(x_{1}, x_{2}, x_{3}, y_{1}, y_{2}\right)$ in game (5) are as following:

$$
\begin{aligned}
& \eta_{1}^{\langle 0\rangle}=\frac{m_{1}}{\sum_{q=1}^{5} m_{q}}=\frac{45^{0.2}}{45^{0.2}+1+15^{-0.2}+27^{-0.2}+9^{0.2}} \approx 0.3697, \\
& \eta_{2}^{\langle 0\rangle}=\frac{m_{2}}{\sum_{q=1}^{5} m_{q}}=\frac{1}{45^{0.2}+1+15^{-0.2}+27^{-0.2}+9^{0.2}} \approx 0.1726, \\
& \eta_{3}^{\langle 0\rangle}=\frac{m_{3}}{\sum_{q=1}^{5} m_{q}}=\frac{15^{-0.2}}{45^{0.2}+1+15^{-0.2}+27^{-0.2}+9^{0.2}} \approx 0.1004
\end{aligned}
$$

Taking into account (24) the point estimate (local priority) of the nominal value of the negative impact force $\mu_{1}^{\langle 0\rangle}$ of variable $y_{1}$ of enterprise for the state gain $P\left(x_{1}, x_{2}, x_{3}, y_{1}, y_{2}\right)$ in game (5): 
$\mu_{1}^{\langle 0\rangle}=\frac{m_{4}}{\sum_{q=1}^{5} m_{q}}=\frac{27^{-0.2}}{45^{0.2}+1+15^{-0.2}+27^{-0.2}+9^{0.2}} \approx 0.0893$

which turns out to be almost three times less than the point estimate (local priority) of the nominal value of the negative impact force $\mu_{2}^{\langle 0\rangle}$ of variable $y_{2}$ for the state gain $P\left(x_{1}, x_{2}, x_{3}, y_{1}, y_{2}\right)$ in this game: $\mu_{2}^{\langle 0\rangle}=\frac{m_{5}}{\sum_{q=1}^{5} m_{q}}=\frac{9^{0.2}}{45^{0.2}+1+15^{-0.2}+27^{-0.2}+9^{0.2}} \approx 0.2679$

We check matrix (21) for expert estimates for consistency. The consistency index is calculated as:

$I_{\text {con }}=\frac{\eta_{1}^{\langle 0\rangle} \sum_{i=1}^{5} m_{l 1}+\eta_{2}^{\langle 0\rangle} \sum_{i=1}^{5} m_{l 2}+\eta_{3}^{\langle 0\rangle} \sum_{i=1}^{5} m_{l 3}+\mu_{1}^{\langle 0\rangle} \sum_{i=1}^{5} m_{l 4}+\mu_{2}^{\langle 0\rangle} \sum_{i=1}^{5} m_{l 5}-5}{5-1} \approx 0.0557$

As we see, $I_{c o n}<0.1$, i.e., expert estimates in matrix (21) are consistent. Therefore, point estimates of the nominal values of the influence forces $(25)-(27)$ of the components of a state pure strategy (1) and the nominal values of the negative influence forces of the variables $y_{1}$ and $y_{2}(28)$ and (29) can be used in the future.

Note that the value of all indicators: $\left\{\operatorname{Sadap} . \text { sat }\left(\eta_{k}\right)\right\}_{k=1}^{3}, \operatorname{Sadap} . \operatorname{sat}\left(\mu_{1}\right)$, Sadap.sat $\left(\mu_{2}\right)$, $\left\{\text { Sadap.ex. }\left(\eta_{k}\right)\right\}_{k=1}^{3}$, Sadap.ex. $\left(\mu_{1}\right)$, Sadap.ex. $\left(\mu_{2}\right)$

before saturation and exceeding the economic norm, one can evaluate so as to obtain quadratic dependencies. Therefore:

$\operatorname{Sadap} . \operatorname{sat}\left(\eta_{k}=2\right)$

Sadap.ex. $\left(\eta_{k}=2\right) \forall k=\overline{1,3}$

and

Sadap.sat $\left(\mu_{1}=2\right)$ and $\operatorname{Sadap} . \operatorname{sat}\left(\mu_{2}=2\right)$

Sadap.ex. $\left(\mu_{1}=2\right)$ and Sadap.ex. $\left(\mu_{2}=2\right)$

So, estimates (13) of the influence forces of the components of a state pure strategy in the game (5) are written as following:

$\eta_{1}\left(x_{1}\right)=0.3697\left(1+x_{1}^{2}\right) \times$

$\times\left[\left(1+x_{1}-0.6\right)^{2} \times \frac{1+\operatorname{sign}\left(x_{1}-0.6\right)}{2}+\frac{1-\operatorname{sign}\left(x_{1}-0.6\right)}{2}\right]$

$\eta_{2}\left(x_{2}\right)=0.1726\left(1+x_{2}^{2}\right) \times$

$\times\left[\left(1+x_{2}-0.6\right)^{2} \times \frac{1+\operatorname{sign}\left(x_{2}-0.6\right)}{2}+\frac{1-\operatorname{sign}\left(x_{2}-0.6\right)}{2}\right]$

$\eta_{3}\left(x_{3}\right)=0.1004\left(1+x_{3}^{2}\right) \times$

$\times\left[\left(1+x_{3}-0.8\right)^{2} \times \frac{1+\operatorname{sign}\left(x_{3}-0.8\right)}{2}+\frac{1-\operatorname{sign}\left(x_{3}-0.8\right)}{2}\right]$

and estimates (18) of the influence forces of variables $y_{1}$ and $y_{2}$ in the game (5):

$$
\begin{aligned}
& \mu_{1}\left(y_{1}\right)=0.0893\left(1+y_{1}^{2}\right) \times \\
& \times\left[\left(1+y_{1}-0.6\right)^{2} \times \frac{1+\operatorname{sign}\left(y_{1}-0.6\right)}{2}+\frac{1-\operatorname{sign}\left(y_{1}-0.6\right)}{2}\right] \\
& \quad \text { and } \\
& \mu_{2}\left(y_{2}\right)=0.2679\left(1+y_{2}^{2}\right) \times \\
& \times\left[\left(1+y_{2}-0.4\right)^{2} \times \frac{1+\operatorname{sign}\left(y_{2}-0.4\right)}{2}+\frac{1-\operatorname{sign}\left(y_{2}-0.4\right)}{2}\right]
\end{aligned}
$$

Therefore, having estimates of the parameters of the kernel (7) of the game model (5) on a single hypercube (6) explicitly (33) - (37), the value of the hypersurface will be:

$$
\begin{aligned}
& P\left(x_{1}, x_{2}, x_{3}, y_{1}, y_{2}, y_{3}\right)=\left(\prod_{k=1}^{3} x_{k}^{\eta_{k}\left(x_{k}\right)}\right)\left(1-y_{1}\right)^{\mu_{1}\left(y_{1}\right)} \times \\
& \times\left(1-y_{2}\right)^{\mu_{2} y_{2}}-\left(x_{1}-0.6\right)^{\eta_{k}\left(x_{k}\right)} \times\left(x_{2}-0.6\right)^{\eta_{2}\left(x_{2}\right)} \times \\
& \times\left(x_{3}-0.8\right)^{\eta_{3}\left(x_{3}\right)} \times\left(y_{1}-0.6\right)^{\mu_{k}\left(y_{k}\right)} \times\left(y_{2}-0.4\right)^{\mu_{2}\left(y_{2}\right)} \times \\
& \times\left(\min \left\{\operatorname{sign}\left(x_{1}-0.6\right), \operatorname{sign}\left(x_{2}-0.6\right), \operatorname{sign}\left(x_{3}-0.8\right)\right\}\right) \times \\
& \times\left(\min \left\{\operatorname{sign}\left(y_{1}-0.6\right), \operatorname{sing}\left(y_{2}-0.4\right)\right\}\right),
\end{aligned}
$$

with degrees (33) - (37) can be obtained using a powerful software MATLAB (Fig. 1). Figure 1 shows the MATLAB generation code, which allows you to visualize the calculation process according to formulas $(33)-(37)$.

In this software, we will also get a solution of the game (5) on a single hypercube (6).

You must first sample the hypercube (6) in each of its five dimensions. Step $h=0.1$ is sufficient for this. So we get:

$$
\frac{1-0}{h}+1=\frac{1}{0.1}+1=11
$$

counts in each dimension of the hypercube (6). Accordingly, the set of pure state strategies as a unit cube (2) will be represented by $11 \times 11 \times 11=1331$ samples, and the set of pure enterprise strategies as a unit cube (4.4) will be represented by $11 \times 11=121$ samples. So, the kernel (38) of the game (5) after hsampling is defined in 161051 points. Of course, it is impossible to see hypersurfaces (38) even after hsampling, however, interesting and important sections of this hypersurface can be visualized by fixing three of its five variables. Of course, among the three variables that are components of a state pure strategy (1), two variables should be fixed, and among the two variables that are components of an enterprise pure strategy (3), one of them is variable.

For any tax burden and a random indicator characterizing the use of shadow operations by an enterprise in the condition of no turbulence in tax exposure and the estimation of the operational processes of state institutions and the functioning of an enterprise without additional costs to protect business, a state does not receive any benefit: $P\left(x_{1}, 0,0, y_{1}, 0\right)<0 \forall x_{1} \in[0 ; 1]$ and $\forall y_{1} \in[0 ; 1]$.

It is worth noting that with the maximum 
turbulence of the tax impact and the assessment of the state's operational processes and the operation of the enterprise with the largest additional costs incurred to protect the business, the picture will be opposite.

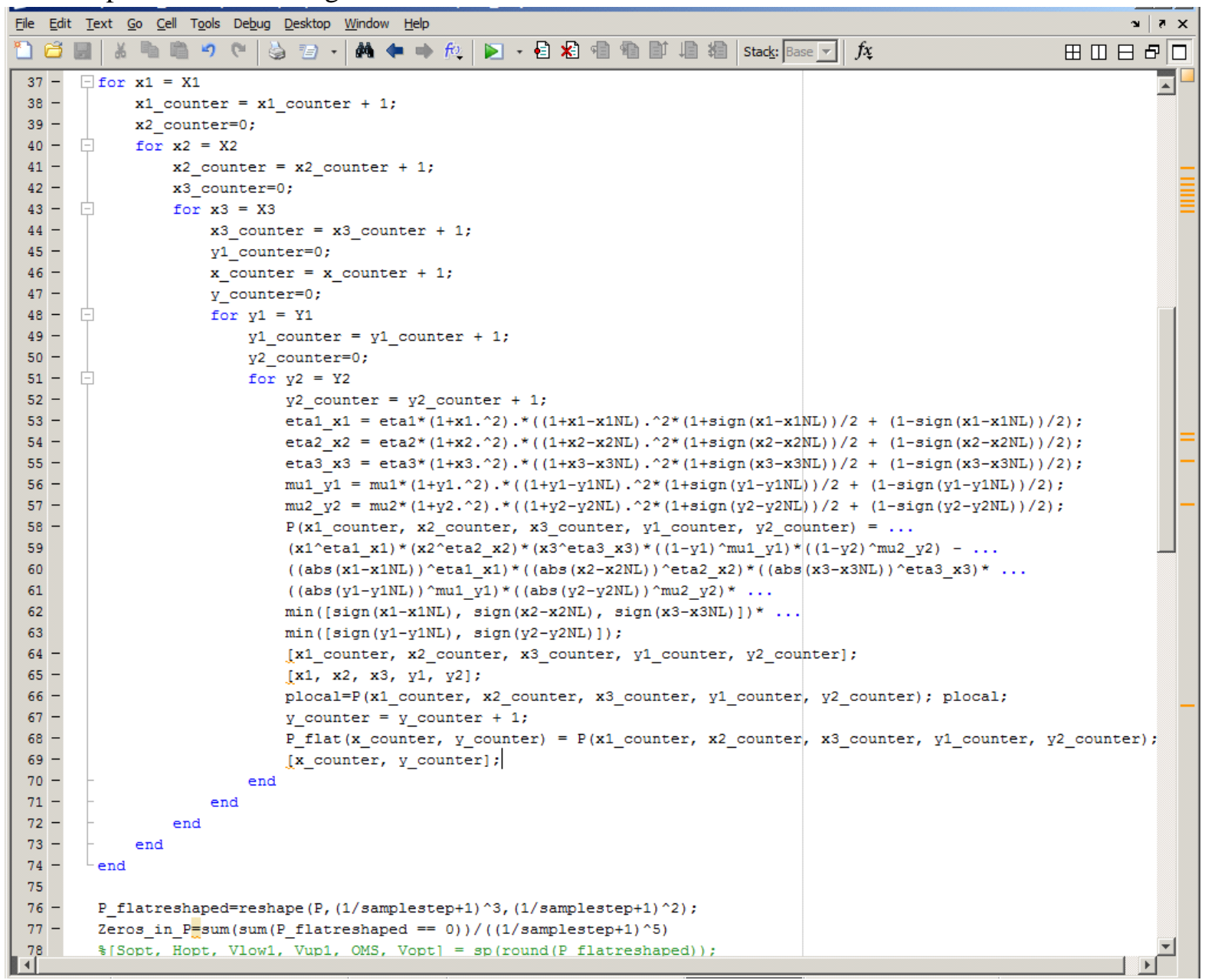

Fig. 1. Code in MATLAB software for calculating and recording values of a sampled hypersurface (38) with degrees $(33)-(37)$

Indeed, from the hypersurface cross section (38) by the tax burden ratio $\mathrm{x}_{1}$ and the indicator characterizing the use of shadow operations by enterprise $y_{1}$ with fixed indicators of tax impact turbulence $\mathrm{x}_{2}=1$ and the assessment of operational processes of government institutions $\mathrm{x}_{3}=1$ and the indicator characterizing additional expenses for protecting the business $\mathrm{y}_{2}=1$ squared values of $\mathrm{x}_{1}$ and $\mathrm{y}_{1}$ there are points $x_{1}^{\langle 0\rangle} \in[0 ; 1]$ and $y_{1}^{\langle 0\rangle} \in[0 ; 1]$, where balance is maintained: $P\left(x_{1}^{\langle 0\rangle}, 1,1, y_{1}^{\langle 0\rangle}, 1\right)=0$.

With the normative limit values of the indicators of tax turbulence and assessment of the state's operational processes according to (19) and the normative limit value of the indicator characterizing the forced additional costs for business protection under (20) conditions, the enterprise almost always loses, moreover, this process is almost uncontrolled for the moderate values of the indicator characterizing the use of shadow operations by the entity. Only by directing this indicator to the maximum value, the company will be able to avoid significant losses.

Approximately the same adverse conditions occur when the maximum values of the indicators of turbulence of the tax impact and the estimation of operational processes and the minimum indicator characterizing the additional costs are required to protect the business. However, even greater losses of the enterprise are likely here with an increase in the tax burden. Then only "entry into shadowing" can reduce these losses. It is interesting that when the tax burden is close to zero, when state losses are significant, the enterprise, by varying the indicator characterizing the use of shadow operations by the enterprise, can affect the usefulness of the state in no way. 
A more complicated situation is when the indicators of tax impact turbulence and the assessment of state's operational processes are minimal, and the indicator characterizing the additional costs for protecting the business is maximized. Here, both a state and an enterprise can lose, but now the enterprise can influence the utility of a state (and its losses, respectively) by varying an indicator characterizing the use by the business entity of almost any value of the tax burden. The only exception is the point $x_{1}=x_{1}^{\text {thereg. lim. }}=0.6$. It is important to note that the company's attempts to reduce losses due to shadowing will be ineffectual, as losses only increase.

With minimal indicators of tax impact turbulence and an assessment of the state's operating processes and a minimum indicator characterizing the use of shadow operations by an enterprise of any tax burden and an arbitrary indicator characterizing additional costs for protecting a business, in the absence of tax turbulence and assessment of the state's operational processes, the state does not receive any benefit:

$$
P\left(x_{1}, 0,0,0, y_{2}\right)<0 \quad \forall x_{1} \in[0 ; 1] \text { and } \forall y_{2} \in[0 ; 1] \text {. }
$$

Note that even the maximum indicators of the tax impact turbulence and the assessment of the state's operational processes with the maximum indicator characterizing the use of shadow operations by the business entity do not guarantee the benefits (positive, of course) for a state $\forall x_{1} \in[0 ; 1]$ and $\forall y_{2} \in[0 ; 1]$.

With the normative limit values of the indicators of tax turbulence and assessing the state's operational processes according to (19) and the normative limit value of the indicator characterizing the use of shadow operations by the business entity according to (20), the company almost always loses again, and this process is almost uncontrollable for moderate values of the indicator characterizing forced additional costs to protect the business. However, if you direct this indicator to the maximum value, the company will probably avoid significant losses, since then the variation of the tax burden by the state will be ineffective.

With the maximum indicators of tax impact turbulence and the assessment of the state's operational processes and a minimum indicator characterizing the use of shadow operations by a business entity, a similar effect is observed, and the enterprise can become relatively profitable even in the absence of shadowing. With a zero tax burden ratio, the company receives additional utility, although it is not able to influence it.
With minimal indicators of the tax impact turbulence and assessment of operational processes, we see that the maximum indicator characterizing the use of shadow operations by a business entity does not guarantee the company to obtain real (positive) benefits $\forall x_{1} \in[0 ; 1]$ and $\forall y_{2} \in[0 ; 1]$. There is also a saddle level at $x_{1}=x_{1}^{\text {thereg. lim }}=0.6$.

With the minimum components of a state pure strategy (1), which together characterize the minimum (passive) tax influence of a state on an enterprise, and with small indicators of shadowing and protective costs, the company still receives real benefits. At the same time directing these indicators to their maximum level, losses of the enterprise increase.

With the maximum components of a state pure strategy (1), which collectively characterize the maximum (aggressive) tax impact of a state on an enterprise.

It is easy to make sure that the game (5) with its kernel (38) is not solved in pure strategies. It is very difficult to prove and analytically solve the game (5) in mixed strategies $\left\{r^{*}(x), q^{*}(y)\right\}$. However, after 0.1 -sampling of the hypersurface (38) instead of the kernel (38), we obtain a five-dimensional matrix:

$\mathrm{P}=\left[P w_{1} w_{2} w_{3} w_{4} w_{5}\right]_{1 \times 1 \times 1 \times 1 \times 11}$

of $11 \times 11 \times 11 \times 11 \times 11$ format with the following elements:

$P w_{1} w_{2} w_{3} w_{4} w_{5}=P\left(\begin{array}{l}0.1\left(w_{1}-1\right), 0.1\left(w_{2}-1\right), \\ 0.1\left(w_{3}-1\right), 0.1\left(w_{4}-1\right), \\ 0.1\left(w_{5}-1\right)\end{array}\right)$

with $w_{q}=\overline{1,11} \forall_{q}=\overline{1,5}$.

Since the first three indices of the element (40) of the matrix (29) correspond to the components of a state pure strategy (1) and the last two indices - to an enterprise pure strategy (3), then the fivedimensional matrix (39) can be reformatted to the ordinary two-dimensional one:

$\overline{\mathrm{P}}=\left[\overline{\mathrm{P}} u v_{5}\right\rfloor_{133 \times 121}$,

of $1331 \times 121$ format with element $\bar{P} u v$, which indexes are:

$u=121 \times\left(w_{1}-1\right)+11 \times\left(w_{2}-1\right)+w_{3}$

with $w_{k}=\overline{1,11} \cdot \forall_{\mathrm{k}}=\overline{1,3}$

$u=11 \times\left(w_{4}-1\right)+w_{5}$ with $w_{j}=\overline{1,11} \cdot \forall_{\mathrm{j}}=\overline{1,2}$

Thus, instead of the game (5), we can consider its approximation in the form of a $1331 \times 121$ matrix game:

$\left\langle\left\{a_{u}\right\}_{u=1}^{1331},\left\{b_{v}\right\}_{v=1}^{121}, \overline{\mathrm{P}}\right\rangle$, 
in which pure strategy $a_{\mathrm{u}}$ of the first player is in line with a state pure strategy:

$x=\left[0.1\left(w_{1}-1\right) 0.1\left(w_{2}-1\right) 0.1\left(w_{3}-1\right)\right]$ for index $(42)$,

and pure strategy $b_{v}$ of the second player is in line with an enterprise pure strategy:

$y=\left[0.1\left(w_{4}-1\right) 0.1\left(w_{5}-1\right)\right]$ for index (43).

As we know, any matrix game always has a solution in pure or mixed strategies. The solution to the game (44) is denoted as:

$\left.\left\{\omega_{o p t}^{\langle x\rangle}\left(a_{u}\right)\right\}_{u=1}^{1331},\left\{\omega_{o p t}^{\langle y\rangle}\left(b_{v}\right)\right\}_{v=1}^{121}\right\}$ in which $\omega_{o p t}^{\langle x\rangle}\left(a_{u}\right)$ is the optimal probability of a state choosing a pure strategy (45), and $\omega_{o p t}^{\langle y\rangle}\left(b_{v}\right)$ is the optimal probability of a state choosing a pure strategy (46).

Figure 2 shows the solution (47) in the form of a screenshot of the MATLAB command window. Figure 2 shows the optimal probabilities for net strategies of government institutions and enterprises for indices of the corresponding type.

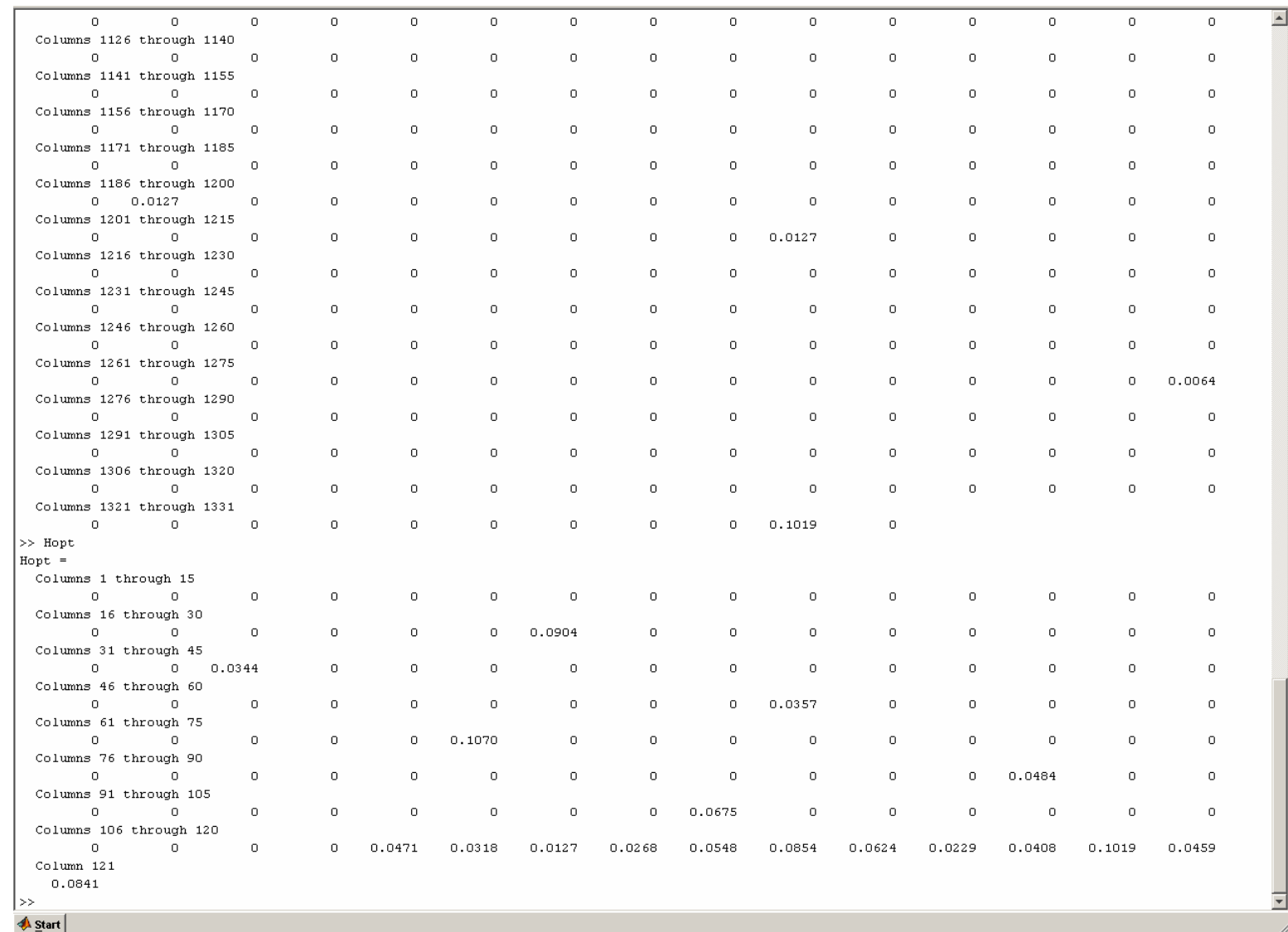

Fig. 2. A screenshot of the MATLAB command window with the solution (47) of matrix game (44)

After 0.1-sampling of the hypersurface (38) the solution of the game (5) in mixed strategies $\left\{r^{*}(x), q^{*}(y)\right\}$ due to the solution (47) we will represent in the following form:

$\left\{\left\{\mathbf{R}_{1}^{*}, \mathbf{R}_{2}^{*}, \mathbf{R}_{3}^{*}\right\},\left\{\mathbf{Q}_{1}^{*}, \mathbf{Q}_{2}^{*}\right\}\right\}$

in which $\mathbf{R}_{k}^{*}=\left[r_{w_{k}}^{*}(k)\right]_{11 \times 1}$ is 11-point approximation of the $k$-th dimension of the probability distribution $r^{*}(x)$, on a single cube (2) with $k=\overline{1,3}$, and $\mathbf{Q}_{j}^{*}=\left[q_{w_{j}}^{*}(j)\right\rfloor_{1 \times 1}$ is 11-point approximation of the $j$-th dimension of the probability distribution $q^{*}(y)$ on a single cube (4) with $j=\overline{1,2}$.
The transition from solution (47) to (48) is carried out according to the following rules. Index for the third component of a state pure strategy (45):

$w_{3}=\chi\left(\frac{u}{11}\right)+11 \times\left(1-\left|\operatorname{sign}\left(\chi\left(\frac{u}{11}\right)\right)\right|\right)$,

in which the function $\chi$ returns the fractional part of its argument. Index for the second component of a state pure strategy (45):

$w_{2}=\chi\left(\frac{\rho\left(\frac{u}{11}\right)}{11}\right)+1$ with $\chi\left(\frac{u}{11}\right) \neq 0$

and 
$w_{2}=\chi\left(\frac{\rho\left(\frac{u}{11}\right)}{11}\right)+11 \times\left(1-\left|\operatorname{sign}\left(\chi\left(\frac{\rho\left(\frac{u}{11}\right)}{11}\right)\right)\right|\right)$ with $\chi\left(\frac{u}{11}\right)=0$,

in which the function $\rho$ returns the whole part of its argument. Index for the first component of a state pure strategy (45):

$w_{1}=\rho\left(\frac{u}{121}\right)+1$ with $\chi\left(\frac{u}{11}\right) \neq 0$ and $\chi\left(\frac{\rho\left(\frac{u}{11}\right)}{11}\right) \neq 0$

and

$w_{1}=\rho\left(\frac{u}{121}\right)$ with $\chi\left(\frac{u}{11}\right)=0$ and $\chi\left(\frac{\rho\left(\frac{u}{11}\right)}{11}\right)=0$

Index for the second component of a pure enterprise strategy (46):

$w_{4}=\chi\left(\frac{u}{11}\right)+11 \times\left(1-\left|\operatorname{sign}\left(\chi\left(\frac{v}{11}\right)\right)\right|\right)$,

and the index for its first component:

$w_{5}=\chi\left(\frac{\rho\left(\frac{v}{11}\right)}{11}\right)+1$ with $\chi\left(\frac{u}{11}\right) \neq 0$

and

$w_{5}=\chi\left(\frac{\rho\left(\frac{v}{11}\right)}{11}\right)+1$ with $\chi\left(\frac{u}{11}\right)=0$

According to the solution (47) of the matrix game (44), in Fig. 2 optimal probabilities for pure state-type strategies (45) for indices of type (42) are the following:

$\omega_{\text {opt }}^{\langle x\rangle}\left(a_{198}\right)=0.0510, \omega_{\text {opt }}^{\langle x\rangle}\left(a_{310}\right)=0.0064, \omega_{\text {opt }}^{\langle x\rangle}\left(a_{427}\right)=0.0446$,

$\omega_{\text {opt }}^{\langle x\rangle}\left(a_{504}\right)=0.0446, \omega_{\text {opt }}^{\langle x\rangle}\left(a_{617}\right)=0.0191, \omega_{\text {opt }}^{\langle x\rangle}\left(a_{696}\right)=0.0255$,

$\omega_{\text {opt }}^{\langle x\rangle}\left(a_{772}\right)=0.0255, \omega_{\text {opt }}^{\langle x\rangle}\left(a_{774}\right)=0.0892, \omega_{\text {opt }}^{\langle x\rangle}\left(a_{792}\right)=0.0127$,

$\omega_{\text {opt }}^{\langle x\rangle}\left(a_{801}\right)=0.3185, \omega_{\text {opt }}^{\langle x\rangle}\left(a_{824}\right)=0.0318, \omega_{\text {opt }}^{\langle x\rangle}\left(a_{856}\right)=0.0127$,

$\omega_{\text {opt }}^{\langle x\rangle}\left(a_{956}\right)=0.1847, \omega_{\text {opt }}^{\langle x\rangle}\left(a_{1187}\right)=0.0127, \omega_{\text {opt }}^{\langle x\rangle}\left(a_{1210}\right)=0.0127$,

$\omega_{\text {opt }}^{\langle x\rangle}\left(a_{1275}\right)=0.0064, \omega_{o p t}^{\langle x\rangle}\left(a_{1330}\right)=0.1019$

moreover

$\omega_{o p t}^{\langle x\rangle}\left(a_{u}\right)=0$ with $u \in\{\overline{1,1331}\} \backslash$

$\{198,310,427,504,617,696,772,774,792$,

$\{801,824,856,956,1187,1210,1275,1330\}$

And the optimal probabilities for pure enterprise strategies of type (46) for indices of type (43) are: $\omega_{\text {opt }}^{\langle y\rangle}\left(b_{22}\right)=0.0904, \omega_{\text {opt }}^{\langle y\rangle}\left(b_{33}\right)=0.0344, \omega_{\text {opt }}^{\langle y\rangle}\left(b_{55}\right)=0.0357$,

$\omega_{\text {opt }}^{\langle y\rangle}\left(b_{66}\right)=0.1070, \omega_{\text {opt }}^{\langle y\rangle}\left(b_{88}\right)=0.0484, \omega_{\text {opt }}^{\langle y\rangle}\left(b_{99}\right)=0.0675$,

$\omega_{\text {opt }}^{\langle y\rangle}\left(b_{110}\right)=0.0471, \omega_{\text {opt }}^{\langle y\rangle}\left(b_{111}\right)=0.0318, \omega_{\text {opt }}^{\langle y\rangle}\left(b_{112}\right)=0.0127$,

$\omega_{\text {opt }}^{\langle y\rangle}\left(b_{113}\right)=0.0268, \omega_{\text {opt }}^{\langle y\rangle}\left(b_{114}\right)=0.0548, \omega_{\text {opt }}^{\langle y\rangle}\left(b_{115}\right)=0.0854$,

$\omega_{\text {opt }}^{\langle y\rangle}\left(b_{116}\right)=0.0624, \omega_{\text {opt }}^{\langle y\rangle}\left(b_{117}\right)=0.0229, \omega_{\text {opt }}^{\langle y\rangle}\left(b_{118}\right)=0.0408$,

$\omega_{\text {opt }}^{\langle y\rangle}\left(b_{119}\right)=0.1019, \omega_{\text {opt }}^{\langle y\rangle}\left(b_{120}\right)=0.0459, \omega_{\text {opt }}^{\langle y\rangle}\left(b_{121}\right)=0.0841$,

moreover

$\omega_{\text {opt }}^{\langle x\rangle}\left(b_{u}\right)=0$ with $u \in\{\overline{1,121}\} \backslash$

$\left\{\begin{array}{l}22,33,55,66,88,99,110,111,112,113, \\ 114,115,116,117,118,119,120,121\end{array}\right\}$

Using expressions (49) - (56) and optimal probabilities (57) - (60), we determine the spectra of the solution (48). For the state, the spectrum of its optimal strategy is the following:

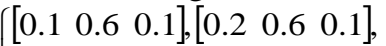

$$
\begin{aligned}
& {\left[\begin{array}{lll}
0.3 & 0.5 & 0.8
\end{array}\right],\left[\begin{array}{lll}
0.4 & 0.1 & 0.8
\end{array}\right] \text {, }} \\
& {\left[\begin{array}{lll}
0.5 & 0.1 & 0
\end{array}\right],\left[\begin{array}{lll}
0.5 & 0.8 & 0.2
\end{array}\right] \text {, }} \\
& \operatorname{supp}\left\{\mathbf{R}_{1}^{*}, \mathbf{R}_{2}^{*}, \mathbf{R}_{3}^{*}\right\}=\left\{\begin{array}{l}
{\left[\begin{array}{lll}
0.6 & 0.4 & 0.1
\end{array}\right],\left[\begin{array}{lll}
0.6 & 0.4 & 0.3
\end{array}\right],} \\
{\left[\begin{array}{llll}
0.6 & 0.5 & 0.1
\end{array}\right],\left[\begin{array}{llll}
0.6 & 0.6 & 0.8
\end{array}\right],}
\end{array}\right. \\
& {\left[\begin{array}{lll}
0.6 & 0.8 & 0.9
\end{array}\right],\left[\begin{array}{lll}
0.7 & 0 & 0.8
\end{array}\right] \text {, }} \\
& {\left[\begin{array}{lll}
0.7 & 0.9 & 0.9
\end{array}\right],\left[\begin{array}{lll}
0.9 & 0.8 & 0.9
\end{array}\right] \text {, }}
\end{aligned}
$$

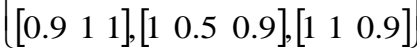

and the corresponding probabilities are (57). For the enterprise, the spectrum of its optimal strategy is the following:

$$
\operatorname{supp}\left\{\left\{Q_{1}^{*}, Q_{2}^{*}\right\}\right\}=\left\{\begin{array}{l}
{\left[\begin{array}{ll}
0.1 & 1
\end{array}\right],\left[\begin{array}{ll}
0.2 & 1
\end{array}\right],\left[\begin{array}{ll}
0.4 & 1
\end{array}\right],\left[\begin{array}{ll}
0.5 & 1
\end{array}\right],\left[\begin{array}{ll}
0.7 & 1
\end{array}\right],} \\
{\left[\begin{array}{ll}
0.8 & 1
\end{array}\right],\left[\begin{array}{ll}
0.9 & 1
\end{array}\right],\left[\begin{array}{ll}
1 & 1
\end{array}\right],\left[\begin{array}{ll}
1 & 0.1
\end{array}\right],\left[\begin{array}{ll}
1 & 0.2
\end{array}\right]} \\
{\left[\begin{array}{ll}
1 & 0.3
\end{array}\right],\left[\begin{array}{ll}
1 & 0.4
\end{array}\right],\left[\begin{array}{ll}
1 & 0.5
\end{array}\right],\left[\begin{array}{ll}
1 & 0.6
\end{array}\right],\left[\begin{array}{ll}
1 & 0.7
\end{array}\right],} \\
{\left[\begin{array}{ll}
1 & 0.8
\end{array}\right],\left[\begin{array}{ll}
1 & 0.9
\end{array}\right],\left[\begin{array}{ll}
1 & 1
\end{array}\right]}
\end{array}\right\},
$$

and the corresponding probabilities are (59). The optimal value of the game (44) is achieved on strategies (61) and (62) with their respective probabilities (57) and (59) is zero. This means that if adhering to the optimal strategy with spectrum (62) and probabilities (59), an enterprise will not waste its surplus resources and the state will not receive surplus resources using the optimal strategy with spectrum (61) and probabilities (57). On the other hand, this means that in the economic system of tax interaction "enterprise - state" a balance is created. According to the principle of optimality, it is not profitable for a state or an enterprise to break this balance.

\section{CONCLUSIONS}

The fundamental basis for modeling the influence of state institutions on the economic security of business entities was analyzed antagonism and at the same time complementarity of the interests of state and business entities and a certain unidirectional 
borderline of the negative impact of government institutions: at each separate moment of time, any state subjects, in accordance with the nature of their activities and tasks, are interested in maximizing the amount of funds that they charge from business entities, however, business entities are interested in minimizing such an amount of funds; institutions and business entities are interested in the long-term activities of business entities; in case if the negative influence of state institutions becomes critical, then, as a minimum, the entities will seek to shade their activities to a maximum, they will simply stop the activity because of its disadvantage.

The basis of the model of the influence of state institutions on the economic security system of business entities was the theory of games. For each of the two participants in the game - state institutions and enterprises - indicators that determine the strategy of the participant in the game and his behavior in such a game are proposed. Each of the proposed indicators' essence is examined and it is shown how the value of such an indicator can be obtained from open sources or calculated. All proposed indicators for both participants in the game are reduced to the same unit scale. The indicators of the model of the antagonistic game between an enterprise and a state are determined; this is the basis for constructing the game field. The content of the game field is determined on the basis of the proposed analytical formula in a multiplicative form.

The developed model of influence of state institutions on the economic security of economic entities is represented by a set of combinations of all possible strategies of behavior of both players in a hypercubic five-dimensional space, which are expressed by the values of the indicators for each of such participants, as well as the calculated "gain" for each of such combinations. Hypercube sampling is performed in 0.1 steps, which allows to consider the content of the model in the two-dimensional matrix $1331^{*} 121$ and in accordance with the task set to simulate the behavior of each participant of the game, justify the choice of strategy for each participant of the game and calculate the expected result ("gain") for such a participant.

The developed model can in fact have both descriptive and prescriptive character, which testifies to its flexibility and versatility - using the developed model of analysis of the influence of state institutions on the system of economic security of domestic enterprises can be evaluated as the actual existing impact in the form of the result of combining the strategies selected by the entity and state institutions on the basis of the proposed indicators, to determine the dynamics of its change (in the form of combining the relevant strategies of the participants in the game in time), and to search for the optimal one by the determined the criterion for the interaction of the participants of the game, accordingly defining the strategies necessary for such interaction of such participants. Additionally, the model may find the use in predicting the impact of public institutions on the economic security of economic entities (based on the analysis of possible strategies of public institutions as a "player" in the constructed game), in predicting the balance of interests of participants in such a game, etc. Despite the mathematical complexity of formalizing the model, it should be emphasized that such complexity is mainly focused on the issues of model development, the calculation of game results in combinations of possible strategies for each player, the search for extremes of the function of the game result, the search for points of balance of interests of all participants of the game - that is, those issues that have already been resolved in the process of creating the model and testing it. Using the same model with the help of code generated in the MATLAB software is not difficult, so the model on this side is complete, ready to use and relatively easy to use (based on generated code) tool, which is of interest in generating the necessary information for both economic entities (for the purpose of assessing or forecasting the impact of state institutions on the economic security of enterprises) and for state regulatory bodies (for the purpose of assessing, modeling and forecasting the effects of regulatory impact on the economy by the state).

Using the model allows to achieve different results depending on the original goal of the study. The main result of using the model is to find the balance of interests in the system "enterprise-state", i.e. the search for those points of stable choice, which violate any of the participants of the game. Partial results that can be obtained from using the developed model are to find a local or general extremum in the game field that will determine the maximum gain of one participant or the total gain of two participants at once; research and quantitative assessment of the actual and maximum possible negative impact of state institutions on the economic security of economic entities; determining the expected change in the impact of public institutions on the economic security of economic entities as a result of changing its strategy; establishment of the best business entity strategy under the existing conditions, which, according to the chosen criterion, will allow ensuring the stable functioning of the economic security system in an enterprise; determining limits of negative influence of state 
institutions on the system of enterprise economic security.

Despite the importance of the obtained results, there are new prospects for further research on the interaction of stakeholders within the socioeconomic systems, which will find a balance of interests of the state and business in the long run.

\section{References:}

[1] Adida, E., DeMiguel V. Supply Chain Competition with Multiple Manufacturers and Retailers. Operations Research, 2011, Vol. 59 (1), pp. 156-172.

[2] Aumann, R. J. Agreeing to Disagree. The Annals of Statistics. Institute of Mathematical Statistics, Vol. 4 (6), 1976. pp. 1236-1239.

[3] Aumann, R. J. Lectures on Game Theory. San Francisco: Westview Press, 1989.

[4] Aumann, R. J. Risk Aversion in the Talmud. Economic Theory. Springer-Verlag, 2003, Vol. 21 (2-3), pp. 233-239.

[5] Bellhouse, D. The Problem of Waldegrave. Journal Électronique d'Histoire des Probabilités et de la Statistique, 2007, Vol. 3 (2), pp. 1-12.

[6] Bruin, B. Game Theory in Philosophy. Topoi, 2005, Vol. 24, pp. 197-208.

[7] Chen, J., Chen, B. Competing with Customer Returns Policies. International Journal of Production Research, 2016, Vol. 54 (7), pp. 2093-2107.

[8] Dixit, A., Nalebuff, B. Thinking Strategically: The Competitive Edge in Business, Politics and Everyday Life. N.Y.: Norton, 1991.

[9] Esmaeili, M., Allameh, G., Tajvidi, T. Using Game Theory for Analysing Pricing Models in Closed-Loop Supply Chain From Short- and Long-Term Perspectives. International Journal of Production Research, 2016, Vol. 54 (7), pp. 2152-2169.

[10] Harsanyi, J. C., Selten, R. A general theory of equilibrium selection in games. Cambridge, Massachusetts: MIT Press, 1988.

[11] Havlovska, N., Savina, H., Davydova, O., Savin, S., Rudnichenko, Y., Lisovskyi, I. Qualitative substantiation of strategic decisions in the field of cost management using the methods of economic mathematical modeling. TEM JOURNAL - Technology, Education, Management, Informatics, 2019, Vol. 8 (3), 959-971.

[12] Hua, M., Lai, I. K. W., Tang, H. Analysis of advertising and a points-exchange incentive in a reverse supply chain for unwanted medications in households based on Game
Theory. International Journal of Production Economics, 2019, Vol. 217(C), pp. 259-268.

[13] Hurwicz, L. The design of mechanisms for resource allocation. The American Economic Review: Papers and Proceedings. American Economic Association via JSTOR, 1973, Vol. 63 (2), pp. 1-30.

[14] Illiashenko, O., Rudnichenko, Ye., Momot, T., Havlovska, N. The enterprise economic security system: the state estimation using management functional types. International Journal for Quality Research, 2020, Vol. 14(1), pp. 183-200.

[15] Maskin, E. Mechanism Design: How to Implement Social Goals. University of California, Irvine School of Social Sciences, 2008.

[16] Mirowski, P. What Were von Neumann and Morgenstern Trying to Accomplish?. In Weintraub, E. Roy (ed.). Toward a History of Game Theory. Durham: Duke University Press, 1992, pp. 113-147.

[17] Myerson, R. B. Game Theory: Analysis of Conflict. Harvard University Press, 1991.

[18] Myerson, R. B. Mechanism Design by an Informed Principal. Econometrica, 1983, Vol. 51 (6), pp. 1767-1797.

[19] Nash, J. F. Equilibrium Points in N-person Games. Proceedings of the National Academy of Sciences of the United States of America, 1950, Vol. 36(1), pp. 48-49.

[20] Nash, J. F., Nagel, R., Ockenfels, A., Selten, R. The agencies method for coalition formation in experimental games. Proceedings of the National Academy of Sciences, 2012, Vol. 109(50), pp. 20358-20363.

[21] Neumann, J. v. The Theory of Games and Economic Behavior. Princeton: Princeton University Press, 1944.

[22] Neumann, J. v. Zur Theorie der Gesellschaftsspiele. Mathematische Annalen, 1928, Vol. 100 (1), pp. 295-320.

[23] Roth, A. E. A Natural Experiment in the Organization of Entry-Level Labor Markets: Regional Markets for New Physicians and Surgeons in the United Kingdom. American Economic Review, 1991, Vol. 81 (3), pp. 415440.

[24] Roth, A. E., Ockenfels, A. Last-minute bidding and the rules for ending second-price auctions: Evidence from eBay and Amazon auctions on the internet. American Economic Review, 2002, Vol. 92 (4), pp. 1093-1103.

[25] Rudnichenko, Ye., Korchevska, L., Mykolaichuk, V., Berezhniuk, I., Havlovska, 
N., Nagorichna, O. Customs qualitative impact on the system of enterprise economic security: modeling and evaluating the results. TEM JOURNAL - Technology, Education, Management, Informatics, 2019, Vol. 8(4), pp. 1176-1184.

[26] Schelling, T. C. Micromotives and Macrobehavior. W.W. Norton \& Company Ltd., 1978.

[27] Schelling, T. C. Some Fun, Thirty-Five Years Ago. Handbook of Computational Economics. Elsevier, 2006, Vol. 2, pp. 1639-1644.

[28] Shapley, L.S. An Example of a Trading Economy with Three Competitive Equilibria. Journal of Political Economy, 1977, Vol. 85, pp. 873-875.

[29] Shapley, L.S. The St. Petersburg Paradox: A Con Game? Journal of Economic Theory, 1977, Vol. 14, pp. 439-442.

[30] Shen, B., Choi, T., Minner, M. S. 2019. A Review on Supply Chain Contracting with Information Considerations: Information Updating and Information Asymmetry. International Journal of Production Research, 2019, Vol. 5457 (15-16), pp. 4898-4936.

[31] Shyian, A. A. Teoriia ihor: osnovy ta zastosuvannia $v$ ekonomitsi ta menedzhmenti. Vinnytsia: VNTU, 2009.

[32] Tirole, J. The theory of industrial organization. Cambridge, Mass. : MIT Press, 1988.

[33] Zermelo's, E. Uber eine Anwendung der Mengenlehre auf die Theorie des Schachspiels, Proc. Fifth Congress Mathematicians, Cambridge University Press, 1913, pp. 501504.

[34] Zlotenko, O., Rudnichenko, Y., Illiashenko, O., Voynarenko, M., Havlovska, N. Optimization of the sources structure of financing the implementation of strategic guidelines for ensuring the economic security of investment activities of an industrial enterprise. TEM JOURNAL - Technology, Education, Management, Informatics 2019, Vol. 8(2), pp. 498-506.

\section{Creative Commons Attribution License 4.0 (Attribution 4.0 International, CC BY 4.0)}

This article is published under the terms of the Creative Commons Attribution License 4.0

https://creativecommons.org/licenses/by/4.0/deed.en_US 